\title{
Confronting the US's Highly Uneven State-by-State Distribution of Diagnostic Radiology Residency Positions
}

\author{
Mohsin Mukhtar MD, Korbin Davis MD, Aaron P. Kamer MD, Richard B. \\ Gunderman MD, PhD
}

Department of Radiology \& Imaging Sciences, Indiana University School of Medicine, 702 North Barnhill Drive, Room 1053, Indianapolis, IN 46202

Key Words: Diagnostic radiology residency positions; Geographic distribution

This is the author's manuscript of the article published in final edited form as:

Mukhtar, M., Davis, K., Kamer, A. P., \& Gunderman, R. B. (2019). Confronting the US's Highly Uneven State-byState Distribution of Diagnostic Radiology Residency Positions. Academic Radiology, 26(11), 1562-1564. https://doi.org/10.1016/j.acra.2019.07.023 
The state-by-state distribution of diagnostic radiology residency positions is important for several reasons. The location in which residents train is positively correlated with their eventual practice location, and states with relatively few residents may have greater difficulty meeting needs for radiology services (1). The presence of residents can also enrich the educational experiences of other learners such as medical students, but this is less likely to happen when residents are in short supply (2). Finally, because residency training programs can promote a culture of inquiry, a dearth of residents may undermine intellectual engagement within a professional community (3).

To determine the degree of uniformity with which residency training programs are distributed across the United States, we used the 2018 National Resident Matching Program database to determine the number of diagnostic radiology residents in each of the 50 US states, dividing the total by US census 2010 data for the population (in millions) of each state. The results are summarized in Figure 1, a map which shows the number of residents per million population in each of the 50 US states.

Figure 1.

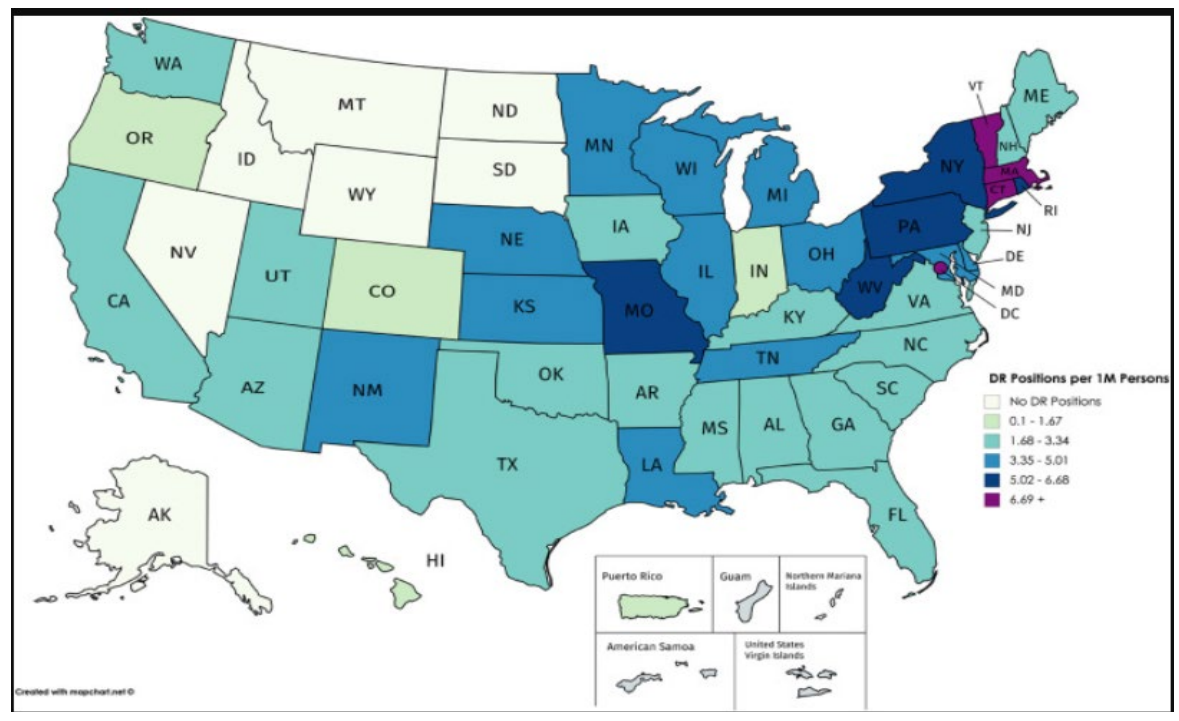

Distribution of diagnostic radiology residency training positions, per 1 million population. 
The mean number of diagnostic radiology residency positions per million is 3.34 , but there is wide variation. For example, seven states have no diagnostic radiology residency positions: Alaska, Idaho, Montana, Nevada, North Dakota, South Dakota, and Wyoming. Some of these states, such as North and South Dakota, have medical schools, but medical students at such schools do not have the opportunity to observe or interact with learners or educators in residency programs.

Five other states have less than one-half the mean number of residents per million population: Hawaii (0.70), Oregon (1.21), Puerto Rico (1.50), Colorado (1.61), and Indiana (1.65). Again, it is likely that students training in such medical schools enjoy fewer opportunities to observe and learn from radiology residents, to benefit directly and indirectly from resident education programs, and to become involved in research and service with faculty members who also teach residents.

By contrast, four states have more than twice the mean number of residents per million population: Washington, DC (11.53), Vermont (9.62), Massachusetts (8.75), and Connecticut (8.08). Such large concentrations of residents could have adverse consequences, such as producing an oversupply of radiologists in such states and undermining the ability of medical students to compete successfully with residents in gaining hands-on experience in radiology.

At a broader level, Figure 1 shows that the lowest density of residents is found in the Rocky Mountain region, where many states have no diagnostic radiology residents. By contrast, the highest concentrations of residents are found in the Northeast region, followed by the Midwest. 
The forces responsible for this heterogeneous distribution of radiology residents are complex and cannot be traced entirely to differences in population density. One key factor is the heterogeneous distribution of residency positions in all medical specialties, which radiology tends to mirror. For example, New York (7.71), Massachusetts (6.60), and Rhode Island (6.14) are capped at relatively high numbers of Medicare-funded GME positions per million population, while Montana (0.16), Idaho (0.22), and Alaska (0.31) have far fewer (4).

Another key factor seems to be the distribution of medical schools - states with large numbers of medical schools tend to have high numbers of residents, while states with few medical schools tend to have few residents. It is only natural that the presence of a medical school would foster a learning environment and commitment to education that would tend to foster residency programs, but while this may explain the dearth of residency positions in some states, it does not justify it.

Another important factor seems to be the size of health centers. In general, states with relatively large health systems have relatively high numbers of residents. Again, this makes sense, since a critical mass of funding, infrastructure, and patient volumes is necessary for training programs to provide an adequate educational experience for residents.

Still another important factor appears to be governmental support for the establishment, growth, and maintenance of residency training programs. States and localities with relatively small budgets are likely to encounter greater challenges in supporting residency training, which require extra staffing and a tolerance for 
inefficiencies in areas such as patient throughput that are not necessarily encountered in environments where resident education is not taking place.

The heterogeneous distribution of residents in any medical field also has important political and economic implications. For example, New York has more residents in all specialties than the 31 states with the fewest residents combined. New York not only receives funding for a much higher number of residents, but it also receives a much higher level of funding per resident, resulting in very large disparities between states. For example, New York receives $\$ 103$ per resident in GME funding, as opposed to only $\$ 20$ in lowa and $\$ 2$ in Montana (5).

The huge concentration of radiology residency positions in the Northeast reflects the history of residency training, which was dramatically expanded at a time when people and money were concentrated there. Since then, the country has expanded westward and southward, without a corresponding redistribution of training positions. As a result, many medical students must leave their home state to pursue residency training.

The question of how to respond to such state-by-state disparities in relative numbers of diagnostic radiology residents is similarly complex. Some observers might argue that geographic uniformity is less important than the quality of training available to residents in each location. For example, the very large academic medical centers in some states may permit a depth and diversity of training experiences that would be difficult for smaller academic and community-based programs to match.

Yet were states with relatively few diagnostic radiology residency positions to start or expand existing residency programs, they might be able to provide distinct benefits to 
their trainees, at least those who accept practice positions in the same state. These might include enabling more residents to train in their home state, matching training experiences to the patient populations residents will later serve during their careers, and enabling residents to prepare for a less subspecialized, more generalist approach to practice.

If the US medical education system were being redesigned from scratch, it would be difficult to imagine that a panel of experts would produce the current distribution map of diagnostic radiology residency positions. From the point of view of equity and meeting the healthcare needs of the populations of each state, the current map could be reasonably described as a maldistribution. Some states are training more residents than they need, others are training fewer, and some are training none at all.

To redress this imbalance would be no easy task. It is unlikely that programs in states with large surpluses of residents would be eager to reduce the size of their programs or shutter them entirely. Likewise, the start-up costs of new residency programs might well seem daunting. Nevertheless, the first step in rebalancing the highly uneven state-by-state distribution of diagnostic radiology residency positions is to recognize that it exists. 


\section{REFERENCES}

1. Association of American Medical Colleges

2. Table C4: physician retention in state of residency training, by last completed GME specialty Rep Residents (2015) https://www.aamc.org/data/448492/c4table.html

3. Snell, L. (2011). The Resident-as-Teacher: It's More Than Just About Student Learning. Journal of Graduate Medical Education, 3(3), 440-441. https://doi.org/10.4300/JGME-D-11-00148.1

4. Zallman, L., Ma, J., Xiao, L., \& Lasser, K. E. (2010). Quality of US Primary Care Delivered by Resident and Staff Physicians. Journal of General Internal Medicine, 25(11), 1193-1197. https://doi.org/10.1007/s11606-010-1456-0

5. Mullan, F., Chen, C., \& Steinmetz, E. (2013). The Geography Of Graduate Medical Education: Imbalances Signal Need For New Distribution Policies. Health Affairs, 32(11), 1914-1921. https://doi.org/10.1377/hlthaff.2013.0545

6. Mullan, Chen, and Steinmetz, Health Aff (Millwood). 2013;32(11):1914-21 